\title{
Hydroxyurea-induced hypersensitivity pneumonitis: A case report and literature review
}

\author{
Harminder S Sandhu MD FRCPC ${ }^{1}$, Penny J Barnes MD FRCPC ${ }^{2}$, Paul Hernandez MDCM FRCPC ${ }^{3}$ \\ ${ }^{1}$ Department of Anesthesia; ${ }^{2}$ Department of Pathology and Laboratory Medicine; \\ ${ }^{3}$ Respirology Division, Department of Medicine, Queen Elizabeth II Health Sciences Centre, \\ Dalhousie University, Halifax, Nova Scotia
}

HS Sandhu, PJ Barnes, P Hernandez. Hydroxyureainduced hypersensitivity pneumonitis: A case report and literature review. Can Respir J 2000;7(6):491-495.

Hydroxyurea is a cytotoxic agent indicated in the treatment of a variety of malignant and nonmalignant conditions. Apart from dose-related bone marrow suppression, this antineoplastic agent is generally well tolerated. This report describes a patient with chronic myeloid leukemia who developed severe pneumonitis within four weeks of beginning therapy with hydroxyurea. Pathological examination of a lung specimen obtained by video-assisted thoracoscopic lung biopsy revealed extensive active alveolar and interstitial inflammation, and poorly formed granulomas. After the cessation of hydroxyurea and treatment with systemic corticosteroids, both clinical and radiological resolution of pneumonitis occurred. Physicians using hydroxyurea must be aware of its potentially life-threatening pulmonary toxicity.

Key Words: Drug-induced pulmonary disease; Hydroxyurea; Hypersensitivity pneumonitis
Pneumonie d'hypersensibilité induite par l'hydroxyurée : Rapport de cas et revue de la littérature

RÉSUMÉ : L'hydroxyurée est un agent cytotoxique indiqué pour le traitement de diverses anomalies malignes et non malignes. Outre la suppression de la moelle osseuse liée à la dose, cet antinéoplasique est en général bien toléré. Cet article décrit le cas d'un patient atteint de leucémie myéloïde chronique qui a développé une pneumonie grave dans les quatre semaines suivant le début d'un traitement à l'hydroxyurée. L'examen anatomopathologique d'un spécimen de biopsie pulmonaire par thoracoscopie assistée par la vidéo a révélé une inflammation alvéolaire et interstitielle active étendue et des granulomes mal formés. Après l'arrêt de l'hydroxyurée et une corticothérapie par voie systémique, on a assisté à une résolution clinique et radiologique de la pneumonie. Les médecins qui utilisent l'hydroxyurée doivent être au courant de cette toxicité pulmonaire potentiellement gravissime.

\begin{abstract}
A number of chemotherapy drugs have been associated with pulmonary toxicity including busulfan, bleomycin, chlorambucil, cyclophosphamide, cytosine arabinoside, melphalan, methotrexate, mitomycin and procarbazine $(1,2)$. Hydroxyurea, a ribonucleotide reductase inhibitor, is indicated in the treatment of a variety of malignant and nonma-
\end{abstract}

lignant conditions (3). Apart from dose-related bone marrow suppression, this antineoplastic agent is generally well tolerated. Other side effects include nausea, vomiting, stomatitis, dysuria, allopecia and rash. In the chronic phase of chronic myeloid leukemia (CML), treatment usually involves using an alkylating agent, such as busulfan, alpha-interferon or 

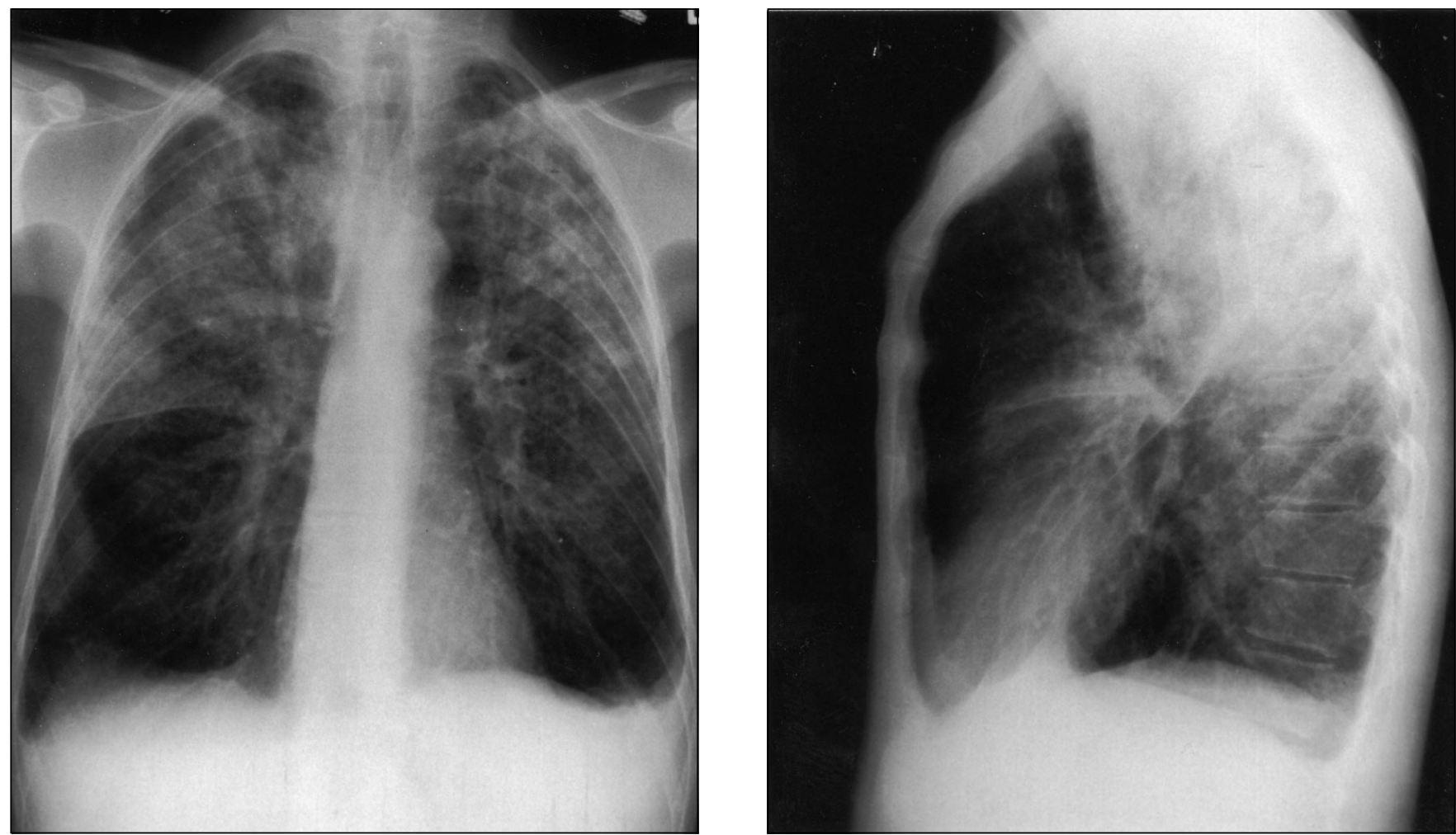

Figure 1) Posteroanterior (left) and lateral (right) chest radiographs showing bilateral upper lobe and superior segment lower lobe airspace and interstitial opacities, and blunting of the costophrenic angles

hydroxyurea (3). Hydroxyurea is increasingly being used for this condition because of its relative lack of serious side effects compared with alternative therapies. We describe a 48-year-old male patient with CML who developed severe pneumonitis within four weeks of beginning therapy with hydroxyurea. The literature relating to acute pulmonary toxicity with hydroxyurea is also discussed.

\section{CASE PRESENTATION}

A 48-year-old man was referred to a hematologist for an increased white blood cell count $\left(230,000 \times 10^{9} / \mathrm{L}\right)$. The patient was married and worked as a carpenter. His only past medical history was community-acquired pneumonia three years earlier. He had a 15 pack-year smoking history. CML was subsequently diagnosed on a bone marrow biopsy and aspirate. The initial treatment included oral hydroxyurea $1000 \mathrm{mg}$ three times daily and oral allopurinol $300 \mathrm{mg}$ daily. In response to near normalization of the white blood cell count and a rise in the platelet count, the dose of hydroxyurea was tapered over two weeks to $500 \mathrm{mg}$ taken orally per day, and allopurinol was discontinued. Eighteen days after the bone marrow biopsy was performed, the patient presented to the emergency department with a four-day history of lethargy, fever, mild shortness of breath, right-sided pleuritic chest pain and left thigh swelling. He was hospitalized on the hematology service with a presumed diagnosis of acute thromboembolic disease (deep vein thrombosis and pulmonary embolism).

On physical examination, the patient was a thin, lethar- gic man in mild respiratory distress. Vital signs revealed a regular pulse of 110 beats/min, respiratory frequency 26 breaths/min, blood pressure $130 / 90 \mathrm{mmHg}$ and an oral temperature $38.5^{\circ} \mathrm{C}$. Jugular venous pressure was not elevated, and there was no peripheral edema in the lower extremities. Precordium examination was unremarkable. There was no chest wall tenderness. Chest was clear to auscultation. There was neither lymphadenopathy nor hepatosplenomegaly. The only clinical evidence of a deep vein thrombosis was a poorly circumscribed area of tenderness and erythema on the inner left thigh approximately $8 \mathrm{~cm}$ in diameter.

Initial laboratory investigations showed a white blood cell count of $16.2 \times 10^{9} / \mathrm{L}$, with a differential of $41 \%$ segmented neutrophils, $25 \%$ basophils, $9 \%$ eosinophils, $7 \%$ lymphocytes, $7 \%$ monocytes and $4 \%$ blasts. Platelet count was $1728 \times 10^{9} / \mathrm{L}$. Hemoglobin was $106 \mathrm{~g} / \mathrm{L}$ with normal red blood cell indices. Serum biochemistry was normal with the exception of mild hyperkalemia. Arterial blood gas values, with the patient breathing room air, were $\mathrm{pH} 7.44$, partial pressure of carbon dioxide $43 \mathrm{mmHg}$, partial pressure of oxygen $68 \mathrm{mmHg}$, oxyhemoglobin saturation $95 \%$ and bicarbonate concentration $28 \mathrm{mmol} / \mathrm{L}$. The electrocardiogram displayed sinus tachycardia with occasional premature ventricular complexes. The chest radiograph showed evidence of small bilateral pleural effusions with minimal right basilar atelectasis. D-dimer assay was negative. Compression Doppler ultrasound performed on both lower extremities showed no evidence of deep vein thrombosis. Ventilation/perfusion lung scan was reported as no significant perfusion defects. 


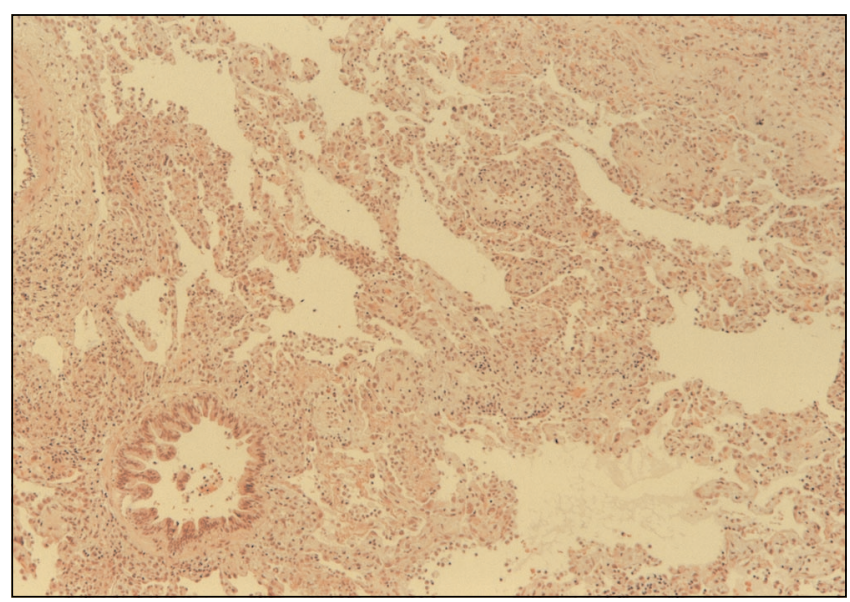

Figure 2) Low power view $(63 \times)$ of the video-assisted thoracoscopic surgery lung biopsy showing extensive and severe airspace and interstitial disease

Once the diagnosis of pulmonary embolism was considered unlikely, the subsequent course in hospital was directed toward elucidating an alternative cause of the presenting symptoms. An infectious etiology was sought under the guidance of an infectious disease consultant. Septic work-up, including urine culture and sensitivity, blood culture and sensitivity, Legionella species urine antigen and serum serology for Legionella species, Mycoplasma pneumoniae and cytomegalovirus, was negative. A whole body gallium scan had findings in keeping with CML but did not suggest a site of infection. Of note, there was no increased uptake of gallium by the lungs.

On the sixth day of hospitalization, a repeat chest radiograph showed new bilateral upper lobe and superior segment lower lobe airspace and interstitial opacities, and bilateral blunting of the costophrenic angles (Figure 1). A respirology consultation was obtained, and a flexible fibreoptic bronchoscopic examination was performed. Visual examination did not indicate any gross abnormality of the vocal cords or tracheobronchial tree. Bronchoalveolar lavage (BAL) samples were obtained from both upper lobes for microbiology and cytology. Washings grew usual respiratory flora but were negative on microscopic examination for Pneumocystis carinii, fungal elements and acid-fast bacilli. Subsequent cultures were negative for fungi, Mycobacterium species, Legionella species and viruses including cytomegalovirus, herpes simplex virus (HSV), adenovirus, influenza virus types $\mathrm{A}$ and $\mathrm{B}$, parainfluenza virus types I, II and III, and respiratory syncytial virus. Cytology of the BAL specimen was unremarkable. No definitive diagnosis was made.

The patient remained symptomatic with fever, dyspnea and pleuritic chest pain. On day 10 of hospitalization, a video-assisted thoracoscopic surgical right upper lobe lung biopsy was performed. Postoperatively, the patient failed extubation in the operating room and again in the postanesthetic care unit because of persistent hypoxemia. A chest radiograph done at that time revealed progression of the upper lobe airspace opacities. The patient was transferred to

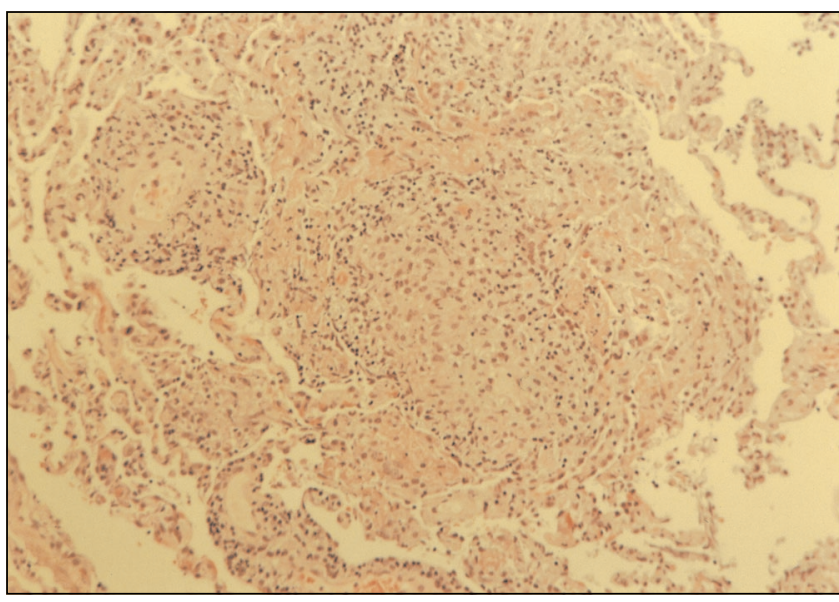

Figure 3) High power view (250×) of the video-assisted thoracoscopic surgery lung biopsy showing a poorly formed granuloma

the intensive care unit and intubated to provide mechanical ventilatory support and high levels of inspired oxygen.

Gram stain results from the lung biopsy specimen indicated the presence of many neutrophils, but no bacteria were identified. The $24 \mathrm{~h}$ and $48 \mathrm{~h}$ bacterial culture reports from the lung biopsy were also negative. On the recommendation of the infectious disease consultant, empirical broadspectrum antibiotic coverage was started (intravenous ceftriaxone $2 \mathrm{~g}$ daily) based on the presence of persistent fever and purulent sputum suctioned from the endotracheal tube. On postoperative day 2 , the patient's condition had improved only slightly. He remained on high levels of ventilatory support but was tolerating a lower inspired fraction of oxygen, down from 1.0 to 0.55 .

Histological sections of the lung biopsy showed extensive and severe airspace, and interstitial disease (Figures 2,3). The interstitium was distorted and expanded by inflammatory cells including neutrophils, histiocytes and a few scattered eosinophils, lymphocytes and plasma cells. Also in the interstitium were aggregations of histiocytic cells, suggesting illdefined granulomas. Airspaces were filled with fibrin and inflammatory cells. Type II pneumocytes were increased in number with prominent nucleoli but without marked atypia or multinucleation. There was no evidence of vasculitis. Chloroacetate esterase staining revealed mature myeloid cells and only rare immature forms; myelogenous leukemic infiltrates were not shown. Special stains for microorganisms were negative including Gram, Gomori methenamine silver, Ziehl-Neelsen, cresyl violet, and immunocytochemical stains for cytomegalovirus, HSV I and HSV II. Viral cytopathic inclusions were not seen. The most likely diagnosis was an acute hypersensitivity drug reaction.

Subsequent management on postoperative day 2 included the discontinuation of hydroxyurea and the initiation of systemic corticosteroid therapy (intravenous solumedrol $160 \mathrm{mg}$ daily in divided doses). The patient defervesced within $24 \mathrm{~h}$ of starting ceftriaxone and solumedrol. Over the next five days in the intensive care unit, the patient's clinical condition 

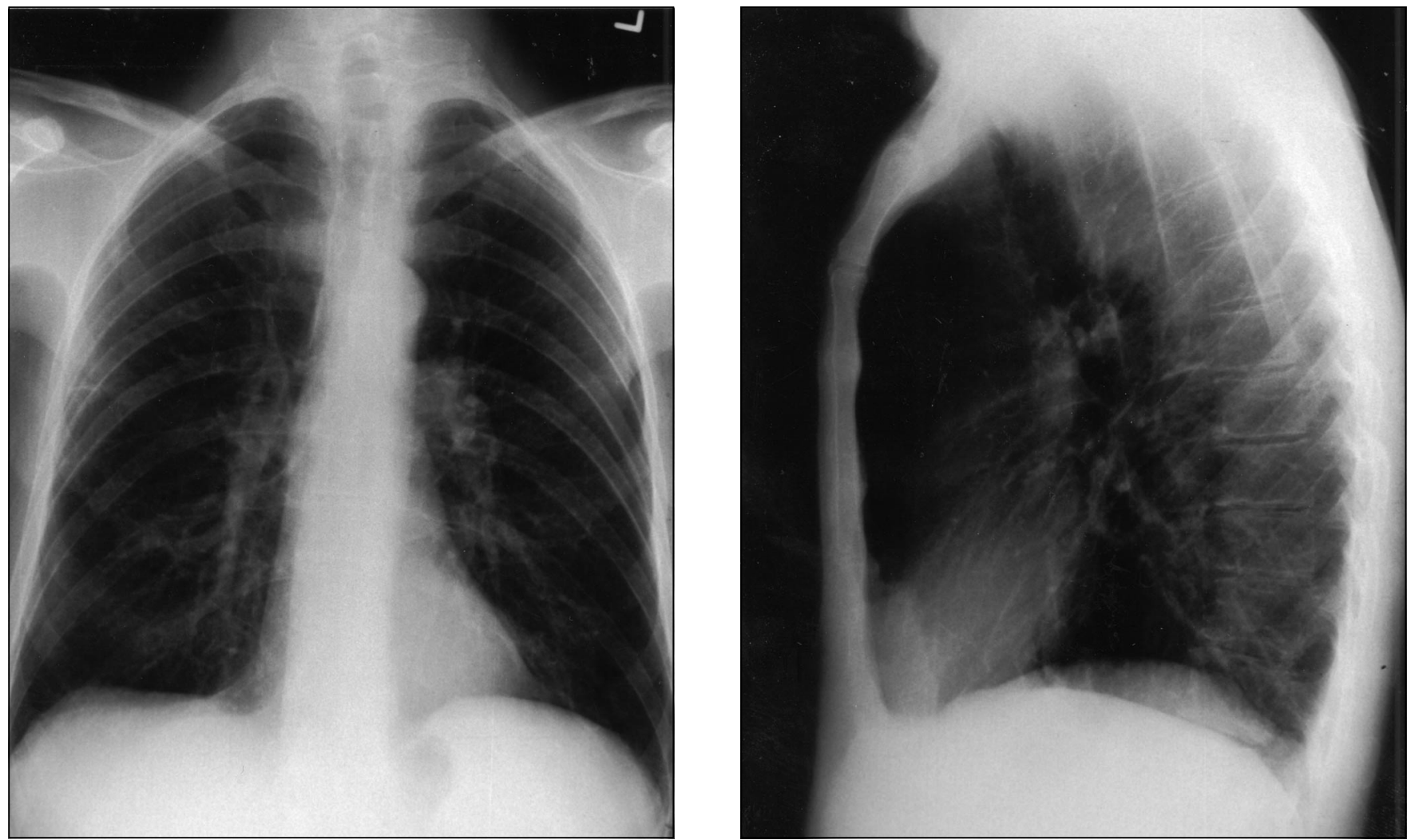

Figure 4) Posteroanterior (left) and lateral (right) chest radiographs showing clearing of multilobar opacities after discontinuation of hydroxyurea and treatment with six weeks of systemic corticosteroids

improved, as did the chest radiograph. He was extubated on postoperative day 7 and transferred to the hematology ward service. On postoperative day 8 , he was no longer dyspneic on mild to moderate exertion and had an oxyhemoglobin saturation of $93 \%$ while breathing room air. By postoperative day 10 , corticosteroids were tapered to oral prednisone $40 \mathrm{mg}$ daily. The patient was started on subcutaneous interferon alpha-2b 2,000,000 units daily for management of CML. He was discharged in good condition on postoperative day 14 on a four-week, tapering course of oral prednisone. Chest radiograph abnormalities had cleared at four weeks after discharge (Figure 4).

\section{DISCUSSION}

The patient showed video-assisted thoracoscopic surgery lung biopsy-proven acute, life-threatening hypersensitivity pneumonitis associated with the treatment of CML with hydroxyurea. Drug-induced pneumonitis is a diagnosis of exclusion. However, the time course of the clinical and radiological progression within four weeks of initiating therapy with hydroxyurea and resolution upon discontinuing the drug and starting systemic corticosteroid therapy is suggestive of this diagnosis. As well, no other etiology could be identified despite extensive investigations.

Four previous case reports in the literature have described acute pneumonitis associated with hydroxyurea (4-7). Jackson et al (4) described a patient who developed fever, dyspnea and bilateral airspace disease as seen on chest radiograph within three weeks of starting oral hydroxyurea $500 \mathrm{mg}$ twice daily for CML. No infectious agent was identified. There was rapid clinical and radiological improvement after the discontinuation of hydroxyurea. Subsequent rechallenge with the drug resulted in the return of symptoms and chest radiograph abnormalities within $24 \mathrm{~h}$. Hydroxyurea was again discontinued, and oral prednisolone $40 \mathrm{mg}$ daily was started. Within four weeks, the patient's clinical and radiological picture had normalized. No lung biopsy was performed.

Kavuru et al (5) described an elderly patient with a myeloproliferative syndrome treated with oral hydroxyurea $500 \mathrm{mg}$ daily. Within 12 weeks of starting hydroxyurea, the patient was hospitalized with fever, dry cough and marked dyspnea. Chest radiograph showed patchy, bilateral interstitial opacities. A transbronchial biopsy performed during bronchoscopy revealed nonspecific interstitial fibrosis and hyperplasia of alveolar lining cells. No infectious etiology was identified. The patient's clinical condition and chest radiograph improved with the discontinuation of hydroxyurea and the institution of oral prednisone $60 \mathrm{mg}$ daily for eight weeks.

A 77-year-old man with myeloproliferative syndrome was treated with hydroxyurea $500 \mathrm{mg}$ twice daily for two weeks (6). He developed fever, lethargy and dyspnea with hypoxia on an arterial blood gas analysis. Chest radiograph showed reticulonodular opacities. No other etiology for his condition was discovered despite bronchoscopy and BAL. There was no improvement with broad spectrum antimicrobial therapy. 
His condition improved dramatically two days after discontinuing hydroxyurea and instituting prednisolone $1000 \mathrm{mg}$ daily.

Four months after receiving the diagnosis of essential thrombocythemia, a 58-year-old man initiated therapy with hydroxyurea $500 \mathrm{mg}$ once daily (7). Four weeks later, the patient developed fever without an obvious cause. Fever was unresponsive to broad spectrum antibiotics but resolved on discontinuation of antibiotics and hydroxyurea. Hydroxyurea was reintroduced four weeks later, and within $24 \mathrm{~h}$, the patient presented to the emergency department with fever, dyspnea, hypoxemia and bilateral interstitial opacities on chest radiograph. No infectious etiology was determined despite extensive cultures including those from BAL fluid. Four days later, antibiotics and hydroxyurea were stopped with a dramatic clinical and radiographical response within another $72 \mathrm{~h}$.

Despite these four published case reports, a number of recent reviews have not listed hydroxyurea among chemotherapeutic agents known to be associated with pulmonary toxicity (1,2,8-11). In addition, the Canadian Adverse Drug Reaction Monitoring Program has received only one report of pneumonitis possibly related to hydroxyurea use between 1965 and 1999 in Canada (unpublished data; Canadian Adverse Drug Reaction Monitoring Program, Bureau of Drug Surveillance, Therapeutic Products Programme, Health Canada).

Drug-induced pulmonary toxicity can result in a variety of histopathological reactions (8-11). Type II pneumocytes often proliferate in response to injury to the alveolar lining cells. Type II pneumocytes themselves may show cytological effects of the offending agent. Other patterns of response are nonspecific interstitial pneumonitis, desquamative interstitial pneumonitis, lymphocytic interstitial pneumonia, eosinophilia, vasculitis, granulomatous inflammation, bronchiolitis obliterans and alveolar proteinosis. These responses, however, are not specific to drug toxicity, and clinical evaluation must be relied upon to exclude accurately conditions that might mimic drug-induced pulmonary disease. The patient in this report had histopathological findings consistent with an acute hypersensitivity drug reaction. Only one prior report of a patient with hydroxyurea-induced acute pulmonary toxicity had lung biopsy material supportive of the diagnosis, which was obtained, in that case, by using transbronchial biopsy during bronchoscopy (5).

\section{SUMMARY}

A patient with CML who developed severe pneumonitis shortly after beginning therapy with hydroxyurea is described. Pathological examination of a lung biopsy specimen revealed extensive active alveolar and interstitial inflammation, and poorly formed granulomas. After the cessation of hydroxyurea and treatment with systemic corticosteroids, there was both clinical and radiological resolution of pneumonitis. Despite the favourable side effect profile of hydroxyurea compared with those of alternative chemotherapeutic agents, physicians using this drug should be aware of its potentially lifethreatening pulmonary toxicity.

ACKNOWLEDGEMENT: The authors thank Iain DC Smith, Drug Information Pharmacist at the Queen Elizabeth II Health Sciences Centre, Halifax, Nova Scotia, for his assistance.

\section{REFERENCES}

1. Twohig KJ, Matthay RA. Pulmonary effects of cytotoxic agents other than bleomycin. Clin Chest Med 1990;11:31-54.

2. Kreisman $\mathrm{H}$, Wolkove N. Pulmonary toxicity of antineoplastic therapy. Semin Oncol 1992;19:508-20.

3. Larson RS, Wolff SN. Chronic myeloid leukemia. In: Lee GR, Foerster J, Lukens J, Paraskevas F, Greer JP, Rodgers GM, eds. Wintrobe's Clinical Hematology, 10th edn. Baltimore: Williams and Wilkins, 1999:2342-73.

4. Jackson GH, Wallis J, Ledingham J, Lennard A, Proctor SJ. Hydroxurea-induced acute alveolitis in a patient with chronic myeloid leukaemia. Cancer Chemother Pharmacol 1990;27:168-9.

5. Kavuru MS, Gadsden T, Lichtin A, Gephardt G. Hydroxyurea-induced acute interstitial lung disease. South Med J 1994;87:767-9.

6. Hennemann B, Bross KJ, Reichle A, Andreesen R. Acute alveolitis induced by hydroxurea in a patient with myeloproliferative syndrome. Ann Hematol 1993;67:133-4.

7. Quintas-Cardama A, Perez-Encinas M, Gonzalez S, Bendana A, Bello JL. Hydroxyurea-induced acute interstitial pneumonitis in a patient with essential thrombocythemia. Ann Hematol 1999;78:187-8.

8. Smith GJ. The histopathology of pulmonary reactions to drugs. Clin Chest Med 1990;11:95-117.

9. Rosenow EC III. Drug-induced pulmonary disease. In: Murray JF, Nadel JA, eds. Textbook of Respiratory Medicine, 2nd edn. Philadelphia: WB Saunders Company, 1994:2117-44.

10. Tanoue LT. Pulmonary toxicity associated with chemotherapeutic agents. In: Fishman AP, ed. Fishman's Pulmonary Diseases and Disorders. New York: McGraw Hill, 1998:1003-16.

11. Fraser RS, Colman N, Muller NL, Paré PD. Fraser and Paré's Diagnosis of Diseases of the Chest, 4th edn. Philadelphia: WB Saunders Company, 1999:2537-83. 


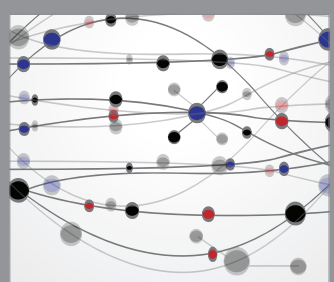

The Scientific World Journal
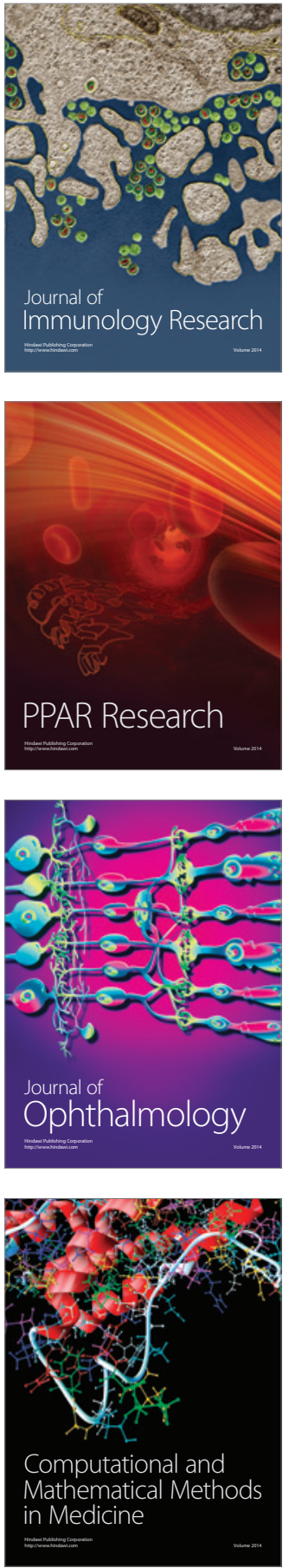

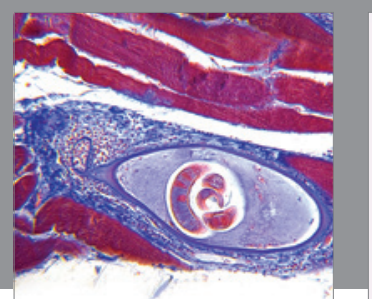

Gastroenterology Research and Practice

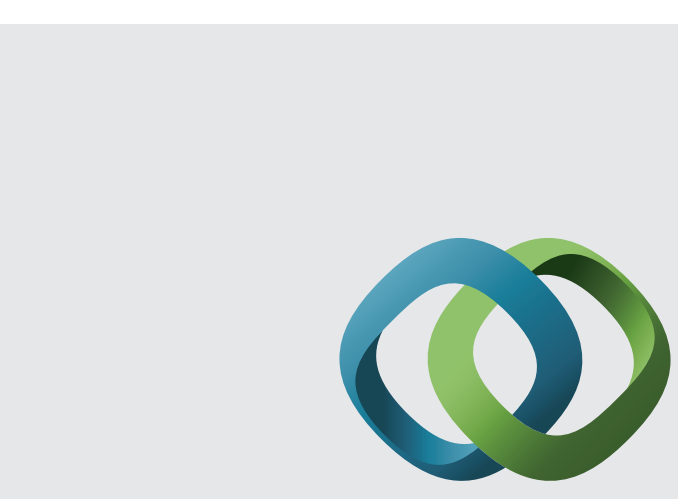

\section{Hindawi}

Submit your manuscripts at

http://www.hindawi.com
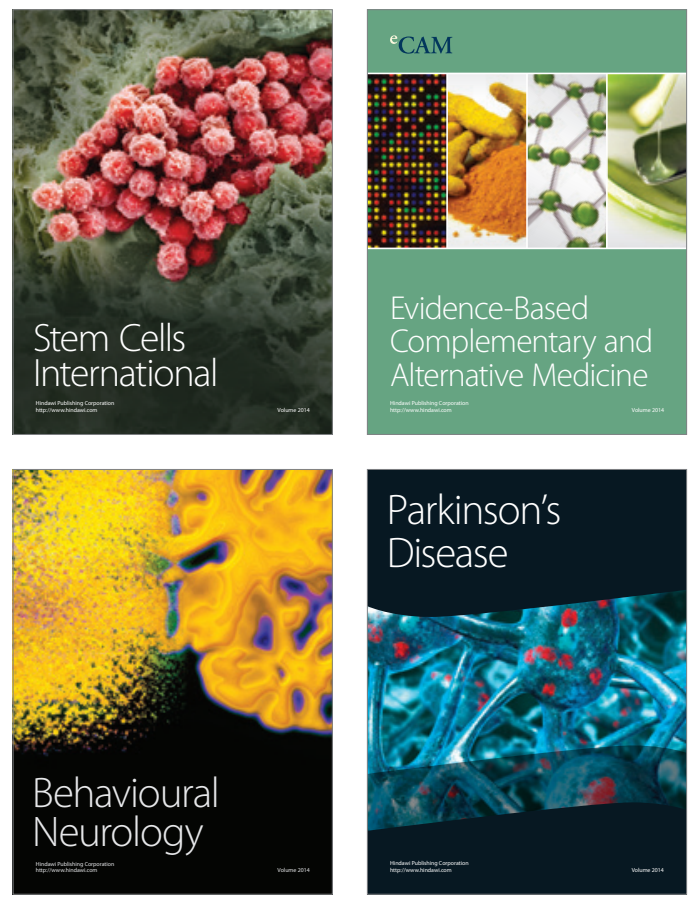
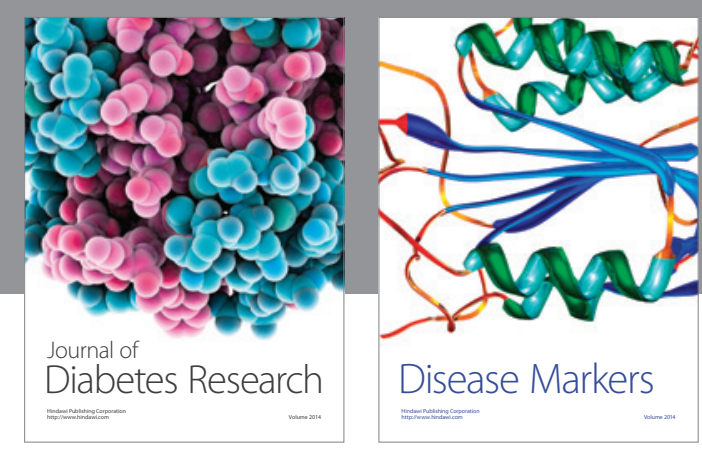

Disease Markers
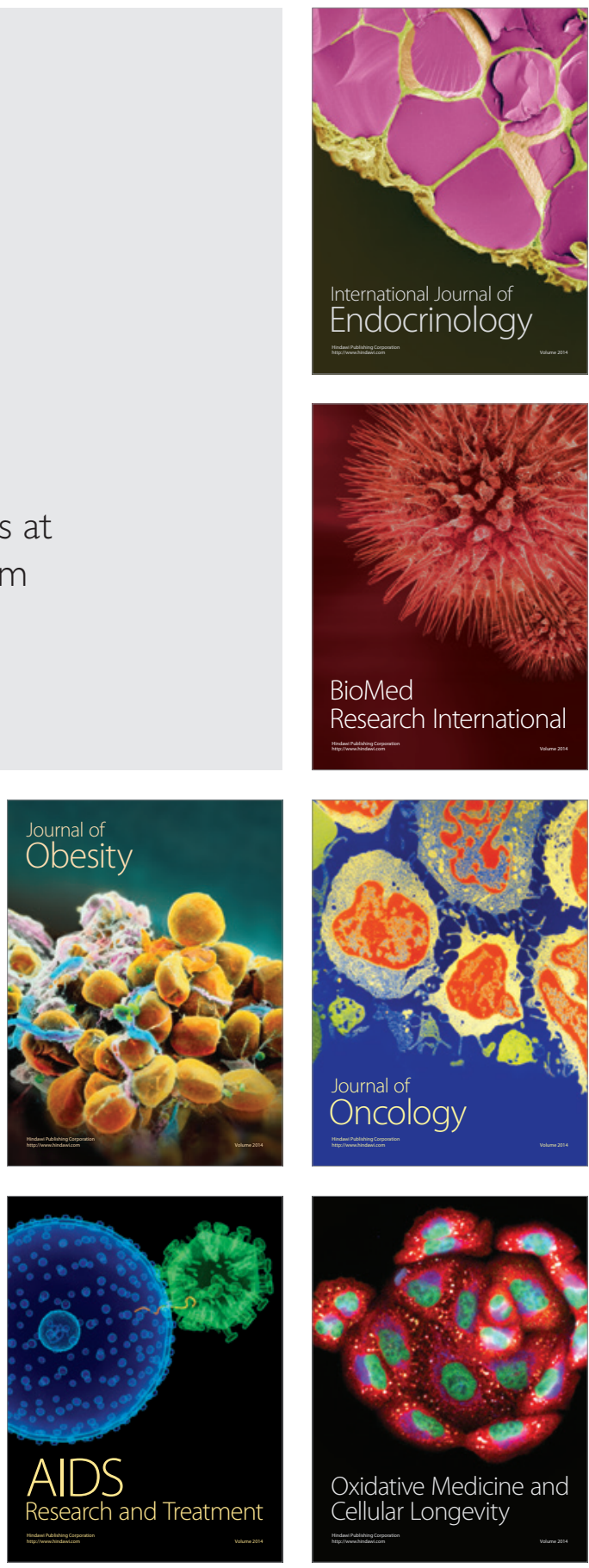